\title{
Impact of Biofilms on Quality of Life of Rhinosinusitis Patients after Endoscopic Sinus Surgery
}

\author{
Lakshmi Vaid, Manish Arya, Neelima Gupta, PP Singh, Rumpa Saha
}

\begin{abstract}
Introduction: The chronic and recalcitrant nature of rhinosinusitis has been known from many years. Many reasons for this have been implicated and biofilms have now been established as one of the cause for its recurrent and persistent nature. Little literature and studies exist confirming this effect. This study presents analysis of sinonasal mucosal samples and correlates presence of biofilms with surgical outcomes.
\end{abstract}

Materials and methods: An analysis of mucosal samples collected during endoscopic sinus surgery from 40 patients of chronic rhinosinusitis (CRS) was done. Preoperative symptoms, endoscopic and radiological scores were documented and mucosal samples collected intraoperatively were sent for biofilm detection. Biofilm detection was performed using microtiter plate method. Postoperatively patients were followed up for minimum of 3 months with endoscopic evaluation and presence of ongoing symptoms was also recorded.

Results: Thirteen patients out of 40 patients showed positive bacterial culture. Eight out of 13 , i.e. $61.53 \%$ bacteria produced biofilms and five out of 13 , i.e. $38.46 \%$ bacteria did not produce biofilms. Patients with biofilms had significantly worse preoperative and postoperative symptom and endoscopic scores. Thus, presence of biofilms was related to poor outcomes.

Conclusion: This study showed that the presence of biofilms was correlated with higher symptom scores and poorer surgical outcomes. Also, more recurrences were found in patients with positive biofilms. This strengthens the belief that biofilms may play an active role in persisting mucosal inflammation and persistent symptoms in some patients of CRS. Treatment modalities aiming removal of biofilms may be important in management of CRS.

Keywords: Biofilm, Rhinosinusitis, Functional endoscopic sinus surgery.

How to cite this article: Vaid L, Arya M, Gupta N, Singh PP, Saha R. Impact of Biofilms on Quality of Life of Rhinosinusitis Patients after Endoscopic Sinus Surgery. Clin Rhinol An Int J 2012;5(3):95-102.

Source of support: Nil

Conflict of interest: None declared

\section{INTRODUCTION}

Chronic rhinosinusitis (CRS) is one of the common medical problems in the world, affecting up to 16 to $20 \%$ of population. Despite the large social impact and economical burden, its pathophysiology remains largely unsolved. ${ }^{1}$

The term rhinosinusitis refers to inflammation of the contiguous tissues of the upper respiratory tract, where insult to the nasal mucosa also affects adjacent sinus tissue. It is agreed that 12 weeks of sinus inflammation is required for a diagnosis of CRS.
Rhinosinusitis may be recalcitrant to treatment, often displaying a chronic relapsing course. To date, there are many proposed theories pertaining to the possible etiological factors. These include the role of superantigens, abnormalities of the inflammatory cytokine cascade, abnormal cell-mediated immune responses, protracted osteitis of the sinus walls and the existence of biofilms.

Biofilms are increasingly being recognized as having an etiological role in CRS. Their extreme resistance to host defence and conventional antibiotic therapy has made the implication of biofilms in the pathogenesis of chronic diseases both attractive and plausible. Recently, biofilms have been implicated in chronic diseases, such as otitis media with effusion, ${ }^{2}$ cholesteatoma, ${ }^{3}$ CRS, ${ }^{4}$ chronic tonsillitis ${ }^{5}$ and in addition on prosthetic devices. ${ }^{6}$

Bacteria existing in a biofilm are surrounded by glycocalyx and grow in a coordinated fashion once a certain density is reached by induction of some signaling molecules. This ability is termed 'quorum sensing'. Such bacteria are less susceptible to antibiotics than the normally existing planktonic bacteria and thus play a role in the chronic and relapsing nature of CRS.?

CRS causes significant physical symptoms and negatively affects quality of life (QOL) and can substantially impair daily function. Subjective and objective parameters used for outcome assessment include SNOT-20, Lund and Mackay grading and Lanza and Kennedy scores. The SNOT-20 asks the patients to rate the severity of their symptoms and social/emotional consequences of their rhinosinusitis. It is scored so that a higher SNOT-20 score indicates worse health related QOL and functional status. ${ }^{8}$ The Lund and Mackay grading system based on a numeric score derived from CT scan is used in the quantification of inflammatory sinonasal disease before surgery. ${ }^{9}$ Endoscopic visualization of sinonasal passage is done and physical findings are scored according to Lanza and Kennedy. ${ }^{10}$

Endoscopic sinus surgery has become the mainstay for CRS. The high success rate, the low incidence of complications and poor response to conservative management has made endoscopic sinus surgery the primary therapy for CRS.

The goal of present study was to find out the impact of biofilms on QOL in cases of CRS having undergone endoscopic sinus surgery. 


\section{MATERIALS AND METHODS}

The study involved retrospective analysis of prospectively collected data in patients of CRS which was conducted for a period of 1 year from January 2011 to December 2011.

Forty patients of CRS were taken. All the patients underwent FESS. The mucosal samples obtained from these patients during surgery were sent for detection of the presence of bacterial biofilms, who were then divided into two groups:

- Group A: Patients with no biofilms detected

- Group B: Patients with positive biofilms.

Patients of CRS were selected after a detailed history; symptoms scored using visual analog scale (VAS) for SNOT-20 questionnaire, anterior rhinoscopy and posterior rhinoscopy, routine blood investigations including absolute eosinophil count, CT paranasal sinuses—axial, coronal and sagittal views, nasal endoscopy and biopsy for histopathology.

Each participant completed the SNOT-20 questionnaire at the preoperative visit. The patients were asked to score a list of 20 symptoms and social and emotional consequences. The list included thick nasal discharge, postnasal discharge, need to blow the nose, facial pain/pressure, sneezing, running nose, cough, ear fullness, ear pain, dizziness, difficulty in falling sleep, getting up at night, lack of good night sleep, waking up tired, fatigue, reduced concentration, reduced productivity, feeling sad, feeling embarrassed and feeling frustrated. Severity of symptoms was graded according to VAS on the scale of 1 to 5 .

Findings on CT scans (Figs 1 and 2) were scored according to Lund and Mackay ${ }^{4}$ scoring system. Scoring was done on the basis of each sinus' opacification and occlusion of the osteomeatal complex.

Findings on endoscopy were scored according to Lanza and Kennedy. ${ }^{10}$ Parameters graded were presence or absence

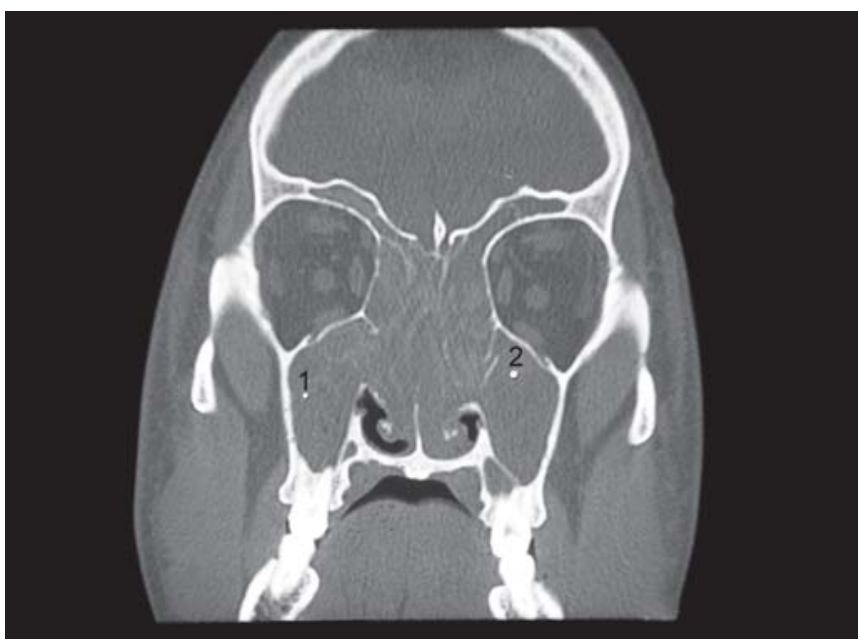

Fig. 1: Coronal cut of CT-PNS showing bilateral polypoidal disease involving maxillary, ethmoids, frontal sinuses and the nasal cavities

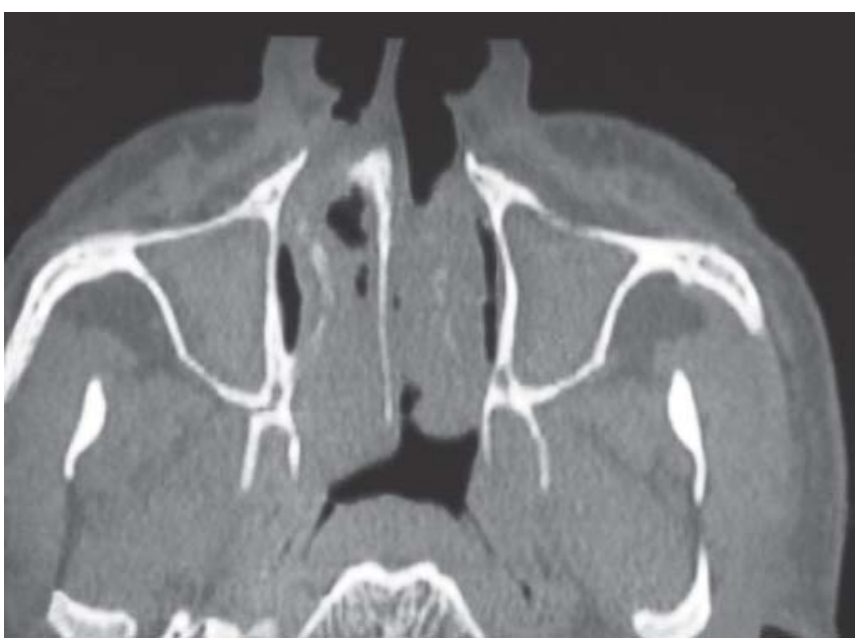

Fig. 2: Axial cut of CT-PNS showing bilateral polypoidal disease involving the maxillary sinuses and the nasal cavities

and extent of nasal polyps, edema, discharge, crusting and scarring.

\section{Surgical Procedure}

Functional endoscopic sinus surgery (FESS) was carried out under general anesthesia. The nasal mucosa was prepared by packing with cotton packs soaked in $4 \%$ xylocaine and 1:15,000 adrenaline solution for 20 minutes. If a septoplasty was needed for either nasal airway obstruction or to gain access, it was performed first. All the patients underwent FESS to clear blockage ensuring complete removal of the disease by using powered instruments (Fig. 3).

Specimens were collected for presence of biofilms or any allergic mucin, fungal debris for identification of fungal hyphae, if present and histopathological examination. Postoperatively, patients were prescribed analgesics and antibiotics for 7 days and the anterior nasal pack was removed on the third postoperative day.

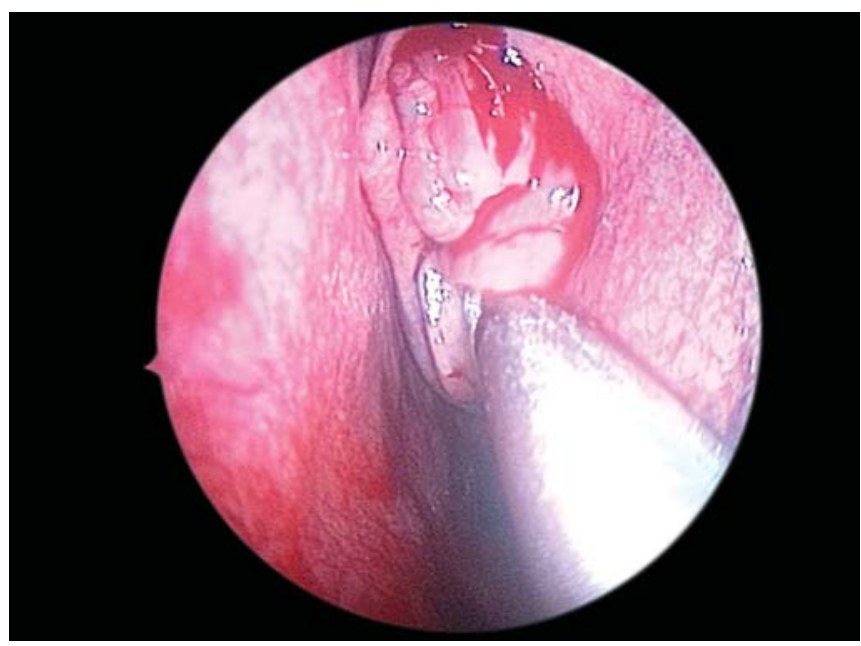

Fig. 3: Endoscopic picture showing polyp removal using microdebrider 
Patient symptoms were rated using SNOT-20 postoperatively after 2 weeks, 1 month and after 3 months in both the groups. Nasal endoscopy was done after 2 weeks, 1 month and 3 months of surgery to look for any granulations, crusts, synechiae and debris. The patency of the ostium was confirmed and the sinus mucosa was examined for any remnant or recurrence of disease.

The data collected was analyzed in terms of details of history, clinical examination, radiological findings and DNE findings before and after surgery. The patients coming with recurrences in terms of reappearance of nasal polyps were identified. Patients with positive bacterial culture and positive bacterial biofilms were identified. A comparison was made between the preoperative and postoperative SNOT and nasal endoscopy scores of patients with positive biofilms and with no biofilms. Patients with positive fungal culture were also identified. Biofilm presence was detected using microtiter plate method described by Christensen et al. ${ }^{11}$

\section{Biofilm Detection}

A total of 40 mucosal or nasal polyps samples were sent in normal saline to Department of Microbiology. Isolates were initially identified by standard microbiological techniques including gram staining and were plated on blood agar, MacConkey agar and Sabouraud's dextrose agar. Ten percent $\mathrm{KOH}$ wet mount was also done to detect any fungal elements. Detection of biofilm was then performed on isolation of a positive bacterial culture using microtiter plate method (Fig. 4). The tissue culture plate (TCP) assay was described by Christensen et al. ${ }^{11}$ It is the most widely used test and is considered as standard test for detection of biofilm formation. TCP method was used with a modification in duration of incubation which was extended to 18 hours.

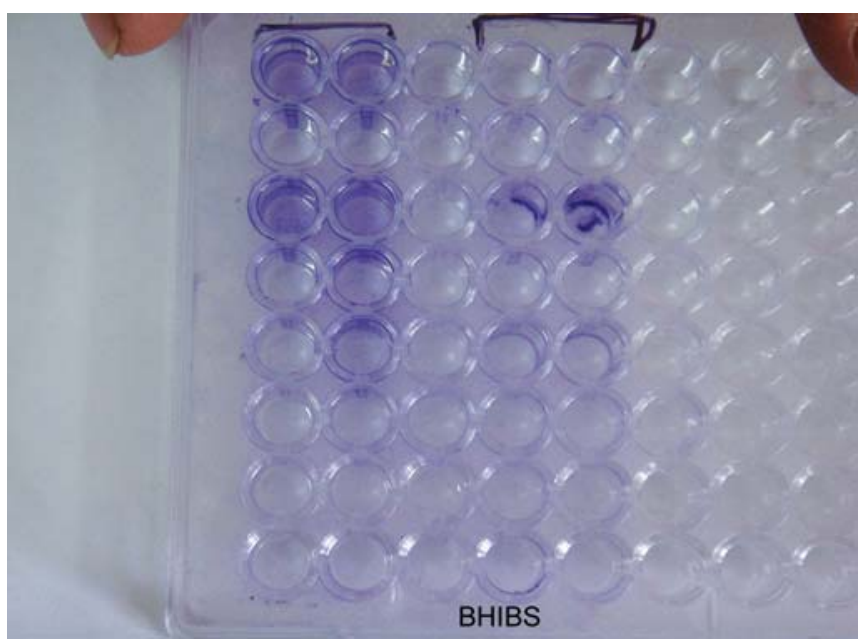

Fig. 4: Microtiter plate showing crystal violet stained biofilm

\section{RESULTS}

Forty patients were included in this study. The average age at the time of presentation was 32.35 years (range, 18-58 years). The study included 19 males and 21 females. Twenty-three patients had unilateral and 17 patients had bilateral disease. Mean preoperative SNOT-20 score was $22.32 \pm 11.46$, with a range of 8 to 48 (Fig. 5) while the mean postoperative SNOT-20 score was $8.2 \pm 7.19$ (Fig. 6).

Raised absolute eosinophilic count was present in only 14 (35\%) cases. The mean preoperative CT score according to Lund and Mackay staging system was $15.87 \pm 7.45$ with a range from 3 to 28. Preoperative diagnostic endoscopy mean score was $8.07 \pm 2.53$ with a range of 3 to 12 .

Histopathological examination showed inflammatory polyps in all the patients.

Thirteen patients out of 40 , i.e. $32.5 \%$ were found to have a positive bacterial culture. Eight out of 13 cultures revealed growth of Staphylococcus aureus out of which four were methicillin resistant. Three cultures grew
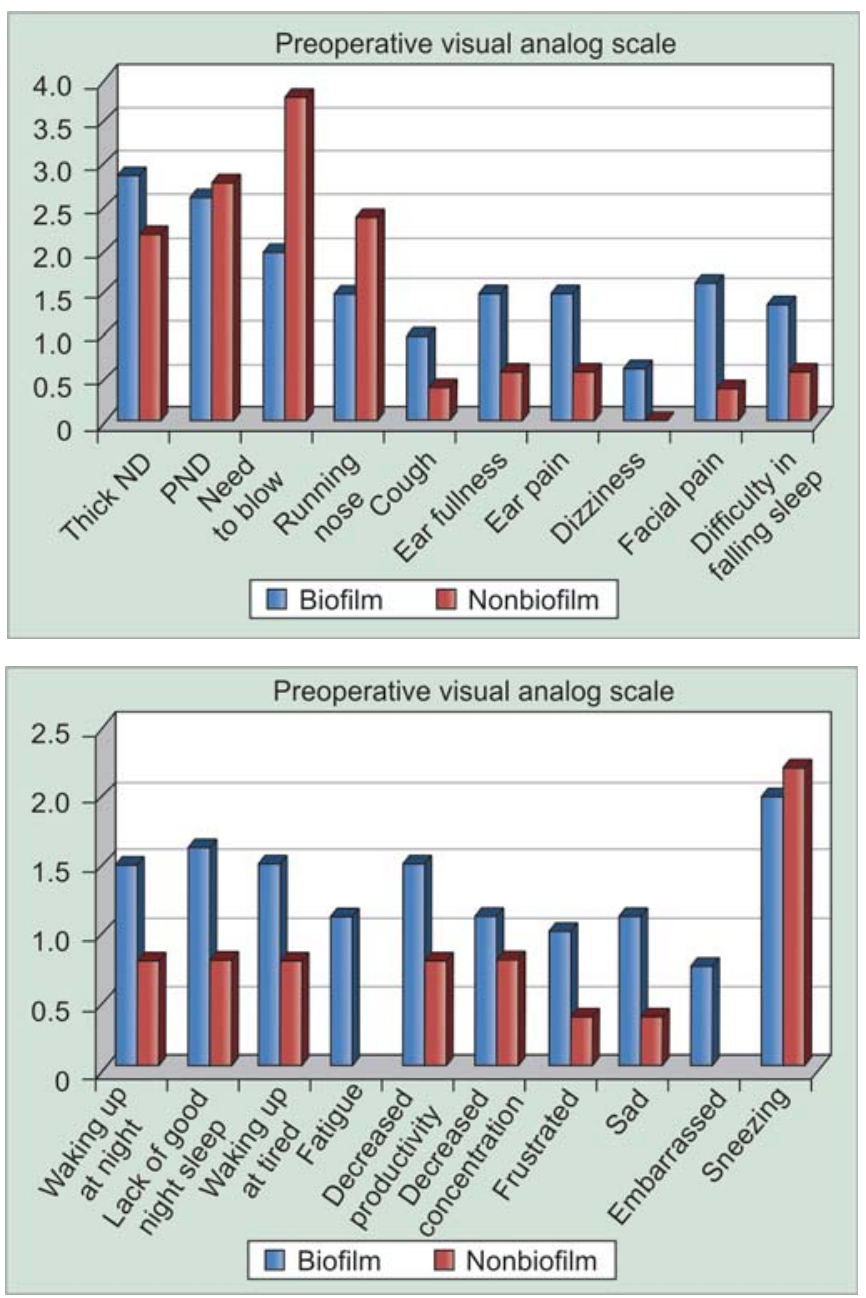

Fig. 5: The preoperative scores comparison between the two groups 


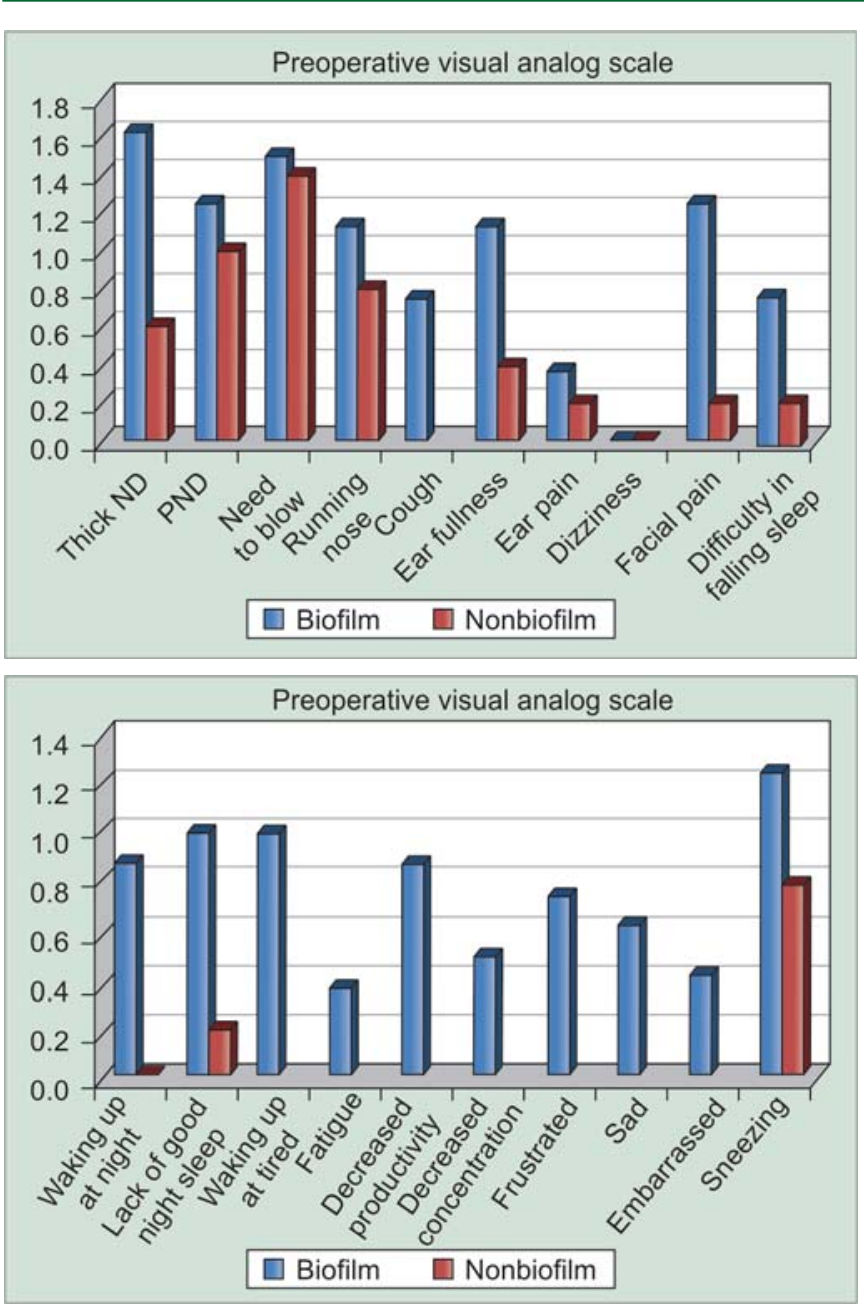

Fig. 6: The postoperative scores comparison between the two groups

Pseudomonas and two were positive for Streptococcus. Proteus showed positive growth in one culture specimens each. One culture was positive for both $S$. aureus and Pseudomonas.
The sensitivity tests of the cultured bacteria were also done, as shown in Table 1. Patients with recurrences were later on put on the specific antibiotics based on the culture sensitivity tests.

Eight patients out of the 13 patients showing positive bacterial culture growth were found to have biofilms present in their samples. In rest of the five patients, no biofilms were detected. Culture rates in biofilm mediated diseases are variable, perhaps dependant on the timing of culture in relation to dispersal of free floating bacteria from the biofilm. This might be the reason all the mucosal samples taken in this study did not yield positive bacterial culture. ${ }^{12}$

Comparison was made between the SNOT-20 and endoscopy scores of the patients having positive biofilms and patients without biofilms out of the patients showing positive bacterial growth.

\section{Postoperative SNOT-20 Symptom Score}

Group A: Patients without biofilms but with positive bacterial culture $(\mathrm{n}=5)$.

This group included only five patients, i.e. $38.46 \%$ of total positive bacterial cultures. There was improvement in most of the nasal symptoms in this group of patients after endoscopic sinus surgery.

Group B: Patients with both positive bacterial culture and positive biofilms $(\mathrm{n}=8)$

This group consisted of eight out of 13 patients, i.e. $61.53 \%$. There was improvement in the nasal symptoms in this group after surgery but it was not as remarkable as improvement shown by patients in group A, as shown in Table 2 and Figure 7.

The comparison between two groups on basis of endoscopy scores is shown in Table 3 and Figure 8.

\begin{tabular}{|c|c|c|c|c|}
\hline S. no. & $\begin{array}{l}\text { Biofilm } \\
\text { status }\end{array}$ & Bacteria & Sensitive & Resistant \\
\hline 1. & Positive & Pseudomonas & Carbenicillin, Meropenem, Ceftazidime, Tazobactam, Amikacin & Polymyxin B \\
\hline 2. & Positive & Pseudomonas & Chloramphenicol, Septran, Imipenem, Ciprofloxacin, Amikacin & Cephalexin \\
\hline 3. & Positive & MSSA & Erythromycin, Ciprofloxacin, Vancomycin, Amikacin, Linezolid & - \\
\hline 4. & Positive & $\begin{array}{l}\text { MSSA, } \\
\text { pseudomonas }\end{array}$ & Clindamycin, Ciprofloxacin, Erythromycin, Vancomycin & - \\
\hline 5. & Positive & Streptococcus & Vancomycin, Linezolid & $\begin{array}{l}\text { Gentamicin, Erythromycin, } \\
\text { Tetracycline, Clindamycin }\end{array}$ \\
\hline 6. & Positive & Streptococcus & Vancomycin & $\begin{array}{l}\text { Gentamicin, Erythromycin, } \\
\text { Tetracycline, Clindamycin, } \\
\text { Linezolid }\end{array}$ \\
\hline 7. & Positive & MRSA & Clindamycin, Vancomycin, Teicoplanin & Gentamicin, ciprofloxacin \\
\hline 8. & Positive & Pseudomonas & Carbenicillin, Meropenem, Ceftazidime, Tazobactam, Amikacin & Polymyxin B \\
\hline 9. & Negative & MSSA & Amikacin, Ciprofloxacin, Linezolid, Vancomycin & Erythromycin \\
\hline 10. & Negative & MRSA & Amikacin, Linezolid, Vancomycin & Ciprofloxacin, Erythromycin \\
\hline 11. & Negative & MSSA & Amikacin, Gentamicin, Clindamycin, Vancomycin & Ampicillin, Ciprofloxacin \\
\hline 12. & Negative & Proteus & - & - \\
\hline 13. & Negative & S. aureus & Vancomycin, Ciprofloxacin, Gentamicin & $\begin{array}{l}\text { Clindamycin, Erythromycin, } \\
\text { Linezolid }\end{array}$ \\
\hline
\end{tabular}




\begin{tabular}{|c|c|c|c|c|c|}
\hline & & Preoperative & 2 weeks & 1 month & 3 months \\
\hline Group A & $\begin{array}{l}\text { Average score } \\
\% \text { of improvement }\end{array}$ & $20.8 \pm 6.94$ & $\begin{array}{l}5 \pm 2.91 \\
75.96 \%\end{array}$ & $\begin{array}{l}3 \pm 2.34 \\
90.38 \%\end{array}$ & $\begin{array}{l}5.8 \pm 4.08 \\
72.11 \%\end{array}$ \\
\hline \multirow[t]{2}{*}{ Group B } & $\begin{array}{l}\text { Average score } \\
\% \text { of improvement }\end{array}$ & $29.875 \pm 6.66$ & $\begin{array}{l}10 \pm 3.96 \\
66.52 \%\end{array}$ & $\begin{array}{l}10.25 \pm 4.23 \\
65.69 \%\end{array}$ & $\begin{array}{l}17.125 \pm 2.03 \\
42.67 \%\end{array}$ \\
\hline & & Preoperative & 2 weeks & 1 month & 3 months \\
\hline Group A & $\begin{array}{l}\text { Average score } \\
\% \text { of improvement }\end{array}$ & $8 \pm 1$ & $\begin{array}{l}4.6 \pm 1.81 \\
42.5 \%\end{array}$ & $\begin{array}{l}3.4 \pm 1.14 \\
57.5 \%\end{array}$ & $\begin{array}{c}3.75 \pm 1.3 \\
53.125 \%\end{array}$ \\
\hline Group B & $\begin{array}{l}\text { Average score } \\
\% \text { of improvement }\end{array}$ & $10 \pm 2$ & $\begin{array}{l}6.375 \pm 2.19 \\
36.25 \%\end{array}$ & $\begin{array}{l}5.25 \pm 1.90 \\
47.5 \%\end{array}$ & $\begin{array}{l}5.375 \pm 2.44 \\
46.25 \%\end{array}$ \\
\hline
\end{tabular}

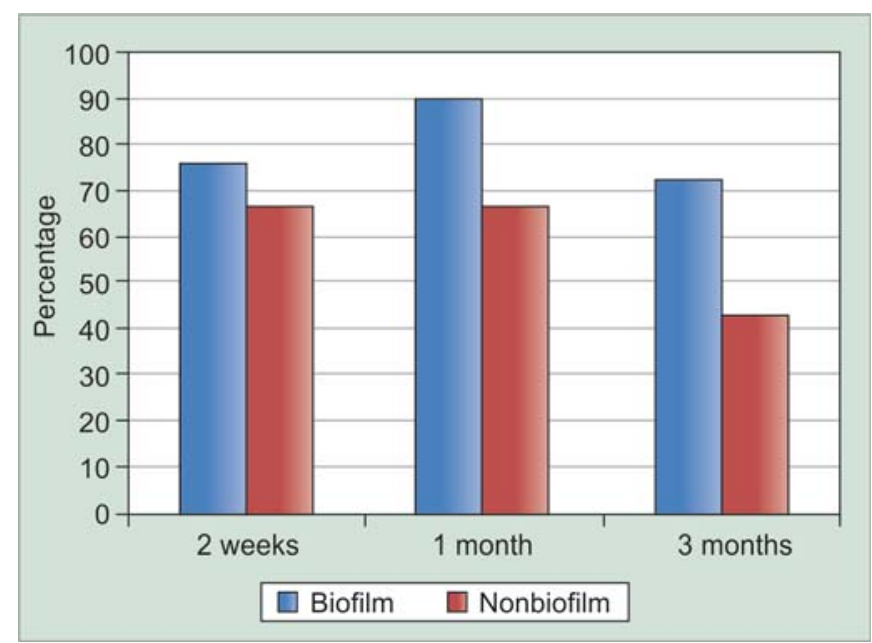

Fig. 7: Comparing the percentage improvement in SNOT-20 scores between the two groups at 2 weeks, 1 month and 3 months

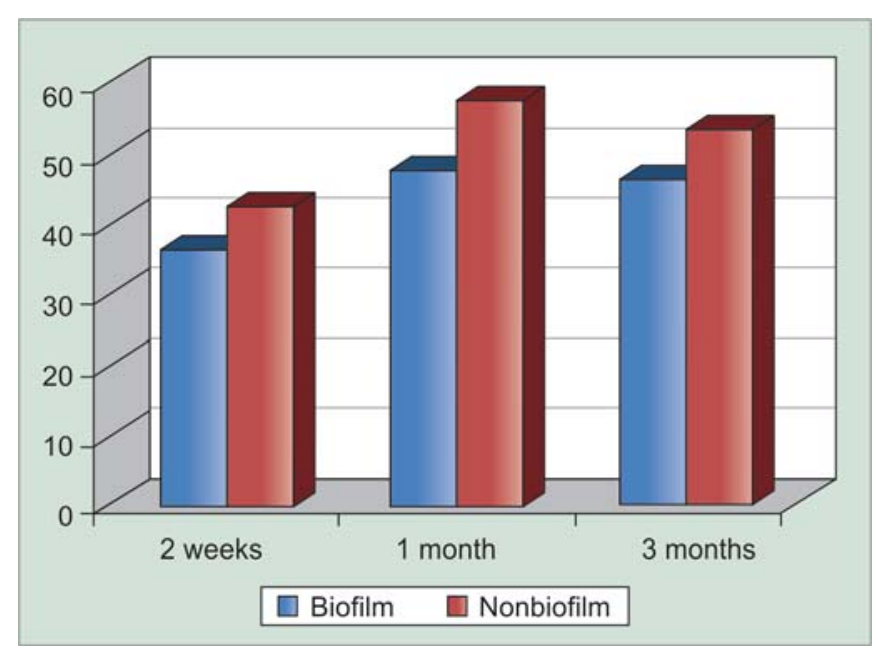

Fig. 8: Comparing the percentage improvement in endoscopy scores between the two groups at 2 weeks, 1 month and 3 months

\section{COMPLICATIONS}

One patient had excessive postoperative bleed requiring nasal packing twice in the postoperative period. Four patients developed synechiae which were divided and resected subsequently during postoperative endoscopy and four patients developed periorbital edema in the postoperative period which relieved spontaneously in few days.

\section{RECURRENCE}

Patients who had recurrence usually presented 3 to 5 months after surgery. A total of $32.5 \%$ cases showed recurrence or persistence of disease. Patients showing recurrence had individual total SNOT-20 score above eight and majority also had a high score on endoscopy.

In group A, i.e. group without biofilms, recurrence was seen in two out of five patients (40\%) whereas in group B, i.e. group with biofilms, recurrence was seen in all the eight out of eight patients (100\%). Three out of the remaining 28 patients showed recurrence that did not show any positive bacterial growth.

Patients having recurrence were put on medical treatment (oral steroids/antibiotics based on the sensitivity tests), minimum for a period of 2 weeks or till symptom free.

Impact on QOL of the patients was also assessed by asking the patients the number of days taken by them to rejoin their normal duties. The average number of days taken by patients to rejoin their normal daily activities was found to be $4.125 \pm 1.15$ with a range of 2 to 7 .

\section{DISCUSSION}

CRS has a profound socioeconomic impact with direct and indirect medical costs, placing a huge financial burden on the society. Despite research efforts into both the medical and surgical management of this condition, there remains a subset of patients who exhibit symptoms refractory to both medical and surgical interventions.

There is emerging evidence to support the case for bacterial biofilms mediating the failure of treatment in such cases, particularly after surgery where there is probable failure to clear all biofilms present within the sinus cavities. 
It is likely that these residual biofilms then go on to mediate a continuing inflammatory reaction leading to disease chronicity and early disease recurrence.

The ability to accurately diagnose the bacterial cause of infection in CRS is fundamental to initiating effective treatment. However, traditional culture based diagnostic microbiology is, in many cases, inadequate and there are conflicting reports outlining specific pathogens associated with CRS.

Biofilm is an organized community of bacteria adherent to an inert or living surface, embedded in a self-produced extracellular polymeric matrix ( $85 \%$ by volume) composed of biopolymers. ${ }^{13,14}$ Biofilm formation is advantageous for the bacteria as it provides protection from both environmental and biological factors. It also provides the bacteria with milieu to exchange genetic information essential for resistance to antimicrobial agents necessary for overall survival. ${ }^{13,15}$

The main aims and objectives in our study were to study the effect of biofilms on the outcome of endoscopic sinus surgeries in patients of CRS and its impact on the QOL. We also tried to find out the clinicopathological correlation in endoscopy, CT findings and symptom scores in patients with and without biofilms.

The average preoperative SNOT-20 score of the eight cases in whose samples biofilms were visualized was 29.8 with a range of 25 to 45 and the median score being 27 . In five cases in whose samples biofilms were not visualized it was 20.8 with the range being 11 to 29 and median score being 23, thus showing that the group with positive biofilms had higher SNOT-20 scores than the group without biofilms. Several reasons have been postulated for these findings. Firstly, biofilm formation is advantageous for the bacteria as it provides protection from factors like temperature, moisture, $\mathrm{pH}$, phagocytosis, host immune system, etc. Secondly, it also provides the bacteria with resistance against the antimicrobial agents.

The group with positive biofilms had a higher preoperative mean endoscopic score of 10 (range, 7-12) while the mean score of the group without biofilm was eight with a range of 7 to 9 .

In a study by Bendouah et al ${ }^{16} 31$ mucosal samples were taken from 19 patients during endoscopic sinus surgery. In this study 22 of 31 samples produced significant biofilms. Biofilm production was noted in 6 of 10 Pseudomonas aeruginosa isolates, 8 of 10 Staphylococcus aureus and 6 of 11 coagulase negative staphylococci. Biofilm formation was associated with a poor postsurgical outcome for Pseudomonas aeruginosa and Staphylococcus aureus but not coagulase negative staphylococci. This showed correlation between biofilm forming capacity of bacteria and unfavorable evolution after FESS.

Similarly in our study biofilm formation was noted by Staphylococcus aureus in three patients, in two patients each by Pseudomonas aeruginosa and Streptococcus and by Gram-negative bacteria in one patient. Out of these, worse postoperative symptom score was seen in cases with biofilms produced by Pseudomonas aeruginosa and Staphylococcus aureus.

Average SNOT-20 score in biofilm group at 2 weeks was $10 \pm 3.96$ with a range of 3 to 14 and in nonbiofilm group it was $5 \pm 2.91$ with a range of 2 to 9 . This slightly worsened to $10.25 \pm 4.23$ (range, 3-18) at 1 month in the biofilm group; while it further improved to $3 \pm 2.34$ (range, 1-7) in the nonbiofilm group. But at 3 months follow-up there was worsening of symptoms in both the groups with the mean score being $17.125 \pm 2.03$ with a range of 14 to 20 in biofilm group and in the nonbiofilm group the score being $5.8 \pm 4.08$ with a range of 1 to 10 . This shows that in the biofilm group, patients experienced $66.52 \%$ improvement in SNOT-20 at 2 weeks with slight worsening to $65.69 \%$ at 1 month and significant worsening to $42.76 \%$ at the end of 3 months. The nonbiofilm group experienced 75.96\% improvement in SNOT-20 at 2 weeks which further improved to $90.38 \%$ improvement at 1 month but worsened to $72.11 \%$ at the end of 3 months. This worsening of symptoms at the end of 3 months could be a reflection of recurrence of formation of polyps in some of the cases. Recurrence was $100 \%$ in cases with positive biofilm while in the nonbiofilm group recurrence was in $40 \%$.

Several authors have suggested that the presence of biofilms is often the predisposing factor for the chronic and recurrent nature of CRS. Their extreme resistance to host defenses and conventional antibiotic therapy has made the implication of biofilms in the pathogenesis of chronic diseases both attractive and plausible. Psaltis et $\mathrm{al}^{17}$ concluded that worsening of symptoms in some patients of CRS after endoscopic sinus surgery could be the result of presence of biofilms in sinonasal mucosa of those patients.

In our study biofilm formation was noted with Staphylococcus aureus in three patients, in two patients each by Pseudomonas aeruginosa and Streptococcus and by Gram-negative bacteria in one patient. Out of these, worse postoperative symptom score was seen in cases with biofilms produced by Pseudomonas aeruginosa and Staphylococcus aureus.

Endoscopy findings in our study also show higher improvement in the nonbiofilm group, i.e. 53.125\% improvement at the end of 3 months than the biofilm positive group which showed improvement of $46.25 \%$. 
This is similar to the study done by Psaltis et $\mathrm{al}^{17}$ in which 15 out of the 20 patients in biofilm group, i.e. $75 \%$ patients showed ongoing inflammatory symptoms while only six out of 20 patients in nonbiofilm group, i.e. 30\% patients showed continuing symptoms.

In our study, it was observed that in both biofilm and nonbiofilm group there was slight fall in percentage improvement of endoscopy score from 1 month to 3 months. It could be explained because of the recurrences noted in all the patients in the biofilm group and in two patients of the nonbiofilm group within a period of 1 to 3 months.

We observed no correlation between preoperative CT grade and overall percentage of improvement of symptoms. However, Psaltis et $\mathrm{al}^{17}$ in their study found patients with biofilms had worse preoperative CT scores as compared to patients without biofilms.

Bacterial biofilms are generally resistant to antimicrobial therapy at levels attainable by oral or intravenous administration. What is needed is novel biofilm disrupting therapies that are not toxic to the mucosa. They should interfere with quorum sensing thereby preventing the proliferation of biofilm disease. Current treatments generally include ventilation of sinus cavities using endoscopic sinus surgery, antimicrobial strategies, biofilm disrupting strategies and limited therapies that target quorum sensing. FESS has shown to be capable of reducing the prevalence of bacterial biofilm but did not eliminate them entirely. ${ }^{18}$

Our study has shown that patients with biofilm have more severe disease preoperatively and show persistence of postoperative symptoms, ongoing mucosal inflammation and infection. This study strengthens the evidence for the role that biofilm may play in recalcitrant CRS. There are not many studies which have correlated the clinicopathological picture in patients with and without biofilm detection in the sinonasal mucosa.

Bacterial biofilms represent one of many possible etiologies for the occurrence, persistence and recurrence of disease in CRS. Both the synergistic effect of multiple organisms and the efficacious treatment of biofilms are areas of extensive investigation which will lead to improvement in the case of CRS patients.

\section{CONCLUSION}

It can be concluded that biofilms have a significant negative impact on patients of CRS. Patients with biofilms have higher symptom scores, worse objective findings and more recurrence as compared to patients without biofilms and patients without biofilms that show more improvement after surgical intervention. Biofilms represented an important influence on pathophysiology of CRS. Further understanding of biofilms interaction and microbial organism behavior will provide us with future treatment modalities for this disease.

\section{REFERENCES}

1. Suh JD, Cohen NA, Palmer JN. Biofilms in chronic rhinosinusitis. Curr Opin Otolaryngol Head Neck Surg 2010 Feb;18(1):27-31.

2. Hall-Stoodley L, Hu FZ, Gieseke A. Direct detection of bacterial biofilms on the middle-ear mucosa of children with chronic otitis media. JAMA 2006;296:202-11.

3. Chole RA, Faddis BT. Evidence for microbial biofilms in cholesteatomas. Arch Otolaryngol Head Neck Surg 2002;128:1129-33.

4. Ha KR, Psaltis AJ, Tan L, Wormald PJ. A sheep model for the study of biofilms in rhinosinusitis. Am J Rhinol 2007;21:339-45.

5. Chole RA, Faddis BT. Anatomical evidence of microbial biofilms in tonsillar tissues: A possible mechanism to explain chronicity. Arch Otlaryngol Head Neck Surg 2003;129:634-36.

6. Costerton JW, Montanaro L, Arciola CR. Biofilm in implant infections: Its production and regulation. Int J Artif Organs 2005;28:1062-68.

7. Ferguson BJ, Stolz DB. Demonstration of biofilm in human bacterial chronic rhinosinusitis. Am J Rhinol 2005;19:452-57.

8. Piccirillo JF, Merritt MG Jr, Richards ML. Psychometric and clinimetric validity of the 20-Item Sino-Nasal Outcome Test (SNOT-20). Otolaryngol Head Neck Surg 2002;126(1):41-47.

9. Lund VJ, Mackay IS. Staging in rhinosinusitis. Rhinology 1993;107:183-84.

10. Lanza DC, Kennedy DW. Adult rhinosinusitis defined. Otolaryngol Head Neck Surg 1997;117:S1-S7.

11. Mathur T, Singhal S, Khan S, Upadhyay DJ, Fatima T, Rattan A. Detection of biofilms formation among the clinical isolates of staphylococci: An evaluation of three different screening methods. Ind J Med Microbiol 2006;24(1):25-29.

12. Foreman A, Jervis-Bardy J, Wormald PJ. Do biofilms contribute to the initiation and recalcitrance of chronic rhinosinusitis? Laryngoscope 2011;121:1085-91.

13. Hall-Stoodley L, Costeton JW, Stoodley P. Bacterial biofilms: from the natural environment to infectious diseases. Mat Rev Microbiol 2004;2:95-108.

14. Costerton W, Veeh R,Shirtliff M, et al. The application of biofilm science to the study and control of chronic bacterial infections. J Clin Invest 2003;112:1466-77.

15. Post JC, Stoodley P, Hall-Stoodley L, Ehrlich GD. The role of biofilms in otolaryngologic infections. Curr Opin Otolaryngol Head Neck Surg 2004;12:185-90.

16. Bendouah Z, Barbeau J, Hamad WA, Desrosiers M. Biofilm formation by Staphylococcus aureus and Pseudomonas aeruginosa is associated with an unfavouable evolution after surgery for chronic sinusitis and nasal polyposis. Otolaryngol Head Neck Surg 2006;134:991-96.

17. Psaltis AJ, Weitzel EK, Ha KR, Wormald PJ. The effect of bacterial biofilms on post sinus surgical outcomes. Am J Rhinol 2008;22:1-6

18. Hai PV, Lidstone C, Wallwork B. The effect of endoscopic sinus surgery on bacterial biofilms in chronic rhinosinusitis. Otolaryngol Head Neck Surg 2010 Mar;142(3 Suppl 1):S27-32. 


\section{ABOUT THE AUTHORS}

\section{Lakshmi Vaid}

Professor, Department of Otorhinolaryngology, University College of Medical Sciences and Guru Teg Bahadur Hospital, New Delhi India

Correspondence Address: F-22, Kirti Nagar, New Delhi-15, India Phone: 09868399551, e-mail: prof.vaid@gmail.com

\section{Manish Arya}

Resident, Department of Otorhinolaryngology, University College of Medical Sciences and Guru Teg Bahadur Hospital, New Delhi India

\section{Neelima Gupta}

Associate Professor, Department of Otorhinolaryngology, University College of Medical Sciences and Guru Teg Bahadur Hospital New Delhi, India

\section{PP Singh}

Professor and Head, Department of Otorhinolaryngology, University College of Medical Sciences and Guru Teg Bahadur Hospital New Delhi, India

\section{Rumpa Saha}

Assistant Professor, Department of Microbiology, University College of Medical Sciences and Guru Teg Bahadur Hospital, New Delhi, India 